\title{
The Role of Single-Subject Brain Metabolic Patterns in the Early Differential Diagnosis of Primary Progressive Aphasias and in Prediction of Progression to Dementia
}

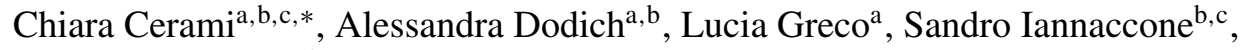 \\ Giuseppe Magnani ${ }^{\mathrm{d}}$, Alessandra Marcone ${ }^{\mathrm{c}}$, Elisabetta Pelagallo ${ }^{\mathrm{c}}$, Roberto Santangelo ${ }^{\mathrm{d}}$, \\ Stefano F. Cappa ${ }^{\mathrm{b}, \mathrm{e}}$ and Daniela Perani ${ }^{\mathrm{a}, \mathrm{b}, \mathrm{f}}$ \\ ${ }^{a}$ Vita-Salute San Raffaele University, Milan, Italy \\ ${ }^{\mathrm{b}}$ Division of Neuroscience, San Raffaele Scientific Institute, Milan, Italy \\ ${ }^{\mathrm{c}}$ Department of Clinical Neuroscience, San Raffaele Turro Hospital, Milan, Italy \\ ${ }^{\mathrm{d}}$ Department of Neurology, San Raffaele Hospital, Milan, Italy \\ ${ }^{\mathrm{e}} N E t S$ Center, Istituto Universitario di Studi Superiori, Pavia, Italy \\ ${ }^{\mathrm{f}}$ Department of Nuclear Medicine, San Raffaele Hospital, Milan, Italy
}

Handling Associate Editor: Christine Bastin

Accepted 28 July 2016

\begin{abstract}
.
Background and Objective: Primary progressive aphasia (PPA) is a clinical syndrome due to different neurodegenerative conditions in which an accurate early diagnosis needs to be supported by a reliable diagnostic tool at the individual level. In this study, we investigated in PPA the FDG-PET brain metabolic patterns at the single-subject level, in order to assess the case-to-case variability and its relationship with clinical-neuropsychological findings.

Material and Methods: 55 patients (i.e., 11 semantic variant/sv-PPA, 19 non fluent variant/nfv-PPA, 17 logopenic variant/lvPPA, 3 slowly progressive anarthria/SPA, and 5 mixed PPA/m-PPA) were included. Clinical-neuropsychological information and FDG-PET data were acquired at baseline. A follow-up of $27.4 \pm 12.55$ months evaluated the clinical progression. Brain metabolism was analyzed using an optimized and validated voxel-based SPM method at the single-subject level.

Results: FDG-PET voxel-wise metabolic assessment revealed specific metabolic signatures characterizing each PPA variant at the individual level, reflecting the underlying neurodegeneration in language networks. Notably, additional dysfunctional patterns predicted clinical progression to specific dementia conditions. In the case of nfv-PPA, a metabolic pattern characterized by involvement of parietal, subcortical and brainstem structures predicted progression to a corticobasal degeneration syndrome or to progressive supranuclear palsy. lv-PPA and sv-PPA cases who progressed to Alzheimer's disease and frontotemporal dementia at the follow-up presented with extended bilateral patterns at baseline.
\end{abstract}

\footnotetext{
${ }^{*}$ Correspondence to: Chiara Cerami, MD, Universitá VitaSalute San Raffaele and San Raffaele Scientific Institute, Division of Neuroscience, Via Olgettina, 60-20134 Milan, Italy.
}

Tel.: +3902 2643 5760; Fax: +3902 2643 5738; E-mail: cerami.chiara@hsr.it. 
Discussion: Our results indicate that FDG-PET voxel-wise imaging is a valid biomarker for the early differential diagnosis of PPAs and for the prediction of progression to specific dementia condition. This study supports the use of FDG-PET imaging quantitative assessment in clinical settings for a better characterization of PPA individuals and prognostic definition of possible endo-phenotypes.

Keywords: FDG-PET, logopenic variant of primary progressive aphasia, non fluent variant of primary progressive aphasia, primary progressive aphasia, positron emission tomography, semantic variant of primary progressive aphasia

\section{INTRODUCTION}

Language networks can be selectively affected by neurodegeneration processes, leading to a progressive language dysfunction, known as primary progressive aphasia (PPA). The possible reasons for this selective susceptibility remain unknown. The condition was first described by Pick. Its rediscovery in the eighties [1] led to an extensive amount of research and to the recognition of a heterogeneous group of conditions with different pathological substrates, in which language impairment is the main symptom at onset that remains the prominent clinical feature during many years of progression [2]. A careful definition of the language phenotype is, together with the recognition of characteristic imaging findings, the best diagnostic predictor of the underlying pathology (see for example [3]).

Recently, a set of criteria has been proposed for the classification of the most common PPA presentations [4]. Three main variants [nonfluent/agrammatic (nfvPPA), semantic (sv-PPA), and logopenic/phonologic (lv-PPA)] are defined on the basis of specific cognitive and linguistic features, and are associated with diffe rent topographical patterns of brain structural or functional changes [5]. After the classification entered into clinical use, it became clear that a number of patients do not neatly fit in any of the three variants (see for example [6,7]), indicating the need for a revision [8].

One of the most challenging obstacles is the differential diagnosis of the PPA variant in the individual patient at an early stage, especially in view of the possible treatment options (e.g., enrollment in clinical trials testing anti-amyloid or anti-tau drugs). For this reason, the contribution of validated neuroimaging tools in the clinical setting is fundamental. In the last 20 years, FDG-PET has gained increasing clinical relevance in supporting differential diagnosis with high accuracy and it is nowadays considered an essential tool for diagnosis in PPAs (e.g., [4]).

In agreement with the different cognitive profiles characterizing the three main PPA variants, FDG-PET imaging patterns significantly differ in these clinical syndromes. The few previous FDG-PET studies were generally based on group analysis, revealing reductions of cerebral glucose metabolism in the left anterior portion of the temporal lobe as the main metabolic signature of sv-PPA [9-12], in the left fronto-insular region and supplementary motor areas in the nfv-PPA [13], and in an extended left temporoparietal network in the lv-PPA variant $[14,15]$.

Despite the above-mentioned typical metabolic signatures in the three PPA syndromes, FDG-PET also revealed the involvement of other brain structures. For example, in sv-PPA, the hypometabolism extended to the limbic structures $[9,16,17]$, the fusiform gyrus [9-11, 18, 19], and the caudate and thalamus $[9,16]$; in lv-PPA the prefrontal cortex is often involved [20, 21]. Very few studies explored functional metabolic changes in PPAs at an individual level $[12,13,22]$, and notably, no study evaluated the role of FDG-PET patterns in PPAs for the prediction of progression to different dementia conditions at follow-up. Current literature is indeed mainly focused in detecting PET signatures (e.g., $[14,15,18]$ ) of AD pathology in PPA cases belonging to the main three PPA syndromes. In the present study, based in a clinical setting, we firstly aimed at investigating the FDG-PET patterns in individual subjects affected by the three main PPA variants $(n=47 ; 11$ sv-PPA, $19 \mathrm{nfv}-\mathrm{PPA}, 17 \mathrm{lv}-\mathrm{PPA}$ ) according to Gorno-Tempini criteria (2011) as well as in a subgroup of unclassified PPA cases $(n=8)$. The second aim was to assess the value early metabolic signatures to predict progression to specific dementia.

The reported studies in PPA variants at group level analysis deserve no consideration to the case-to-case clinical and metabolic heterogeneity in the presentation that indeed could support diagnosis and early signatures of risk of progression to specific dementia conditions, with clinical and research benefit.

\section{METHODS}

\section{Participants}

The retrospective sample includes subjects belonging to the database of the Neurology Centers for 
Table 1

Demographic and clinical features of PPA cases

\begin{tabular}{|c|c|c|c|c|c|c|}
\hline & sv-PPA $n=11$ & nfv-PPA $n=19$ & lv-PPA $n=17$ & SPA $n=3$ & m-PPA $n=5$ & All sample \\
\hline Female/Male ratio & $8 / 3$ & $13 / 6$ & $10 / 7$ & $2 / 1$ & $4 / 1$ & $35 / 20$ \\
\hline $\begin{array}{l}\text { Age in years } \\
\quad(\text { mean } \pm \text { st.dev. })\end{array}$ & $65.82 \pm 7.87$ & $67.42 \pm 8.5$ & $70.71 \pm 7.12$ & $72.33 \pm 4.72$ & $66.4 \pm 7.23$ & $68.29 \pm 7.73$ \\
\hline Age range in years & $56-85$ & $50-85$ & $52-78$ & $67-76$ & $58-77$ & $50-85$ \\
\hline $\begin{array}{l}\text { Years of education } \\
\text { (mean } \pm \text { st.dev. })\end{array}$ & $10.1 \pm 3.48$ & $11.32 \pm 5.09$ & $11.65 \pm 5.17$ & $16.33 \pm 2.89$ & $10.4 \pm 5.32$ & $11.45 \pm 4.70$ \\
\hline $\begin{array}{l}\text { Time from symptoms' } \\
\text { onset in months } \\
\text { (mean } \pm \text { st.dev.) }\end{array}$ & $35.64 \pm 15.84$ & $25.89 \pm 12.2$ & $27 \pm 15.58$ & $32 \pm 6.93$ & $28.8 \pm 6.57$ & $28.78 \pm 13.67$ \\
\hline Positive familiar anamnesis & 1 & 3 & 2 & 2 & 1 & 9 \\
\hline $\begin{array}{l}\text { MMSE adjusted score at } \\
\text { baseline (mean } \pm \text { st.dev.) }\end{array}$ & $23.25 \pm 5.24$ & $20.07 \pm 6.91$ & $21.11 \pm 4.68$ & $26.75 \pm 1.84$ & $20.57 \pm 1.41$ & $21.5 \pm 5.58$ \\
\hline Follow-up time in months & $36 \pm 16.9$ & $18 \pm 8.4$ & $27 \pm 12.7$ & $18.33 \pm 9.45$ & $32.4 \pm 16.76$ & $27.4 \pm 12.55$ \\
\hline
\end{tabular}

(mean \pm st.dev.)

Diagnosis at the follow-up 5 sv-PPA, 6 FTD 5 nfv-PPA, 11 CBD, 3 PSP 7 lv-PPA, 10 AD 2 SPA, 1 CBD 2 AD, 3 FTD

sv-PPA, semantic variant of primary progressive aphasia; nfv-PPA, non fluent variant of primary progressive aphasia; lv-PPA, logopenic variant of primary progressive aphasia; SPA, slowly progressive anarthria; m-PPA, mixed primary progressive aphasia; MMSE, Mini-Mental Status Examination.

Cognitive Disorders of San Raffaele Hospital (Milan, Italy) referred as suspected PPA to the Nuclear Medicine Department of San Raffaele Hospital for an FDG-PET scan in the years between 2011 and 2015. Clinical information (medical history, neurological examination, and neuropsychological assessment) was evaluated by three neurologists (S.I., G.M., A.M.), experts in dementia diagnosis who examined the medical records related to the entire clinical course of each subject from the initial clinical diagnosis to clinical progression. A genetic screening for autosomic dominant mutations was performed in the whole sample. One sv-PPA patient carried a GRN mutation [23]. Patients with signs or symptoms of motor neuron disease were excluded. A family history of neuropsychiatric conditions was reported by the $13 \%$ of patients. Conventional MRI was acquired in each case to exclude the presence of white matter hyperintensities and lacunes of presumed vascular origin.

We included 47 cases classified as sv-PPA $(n=11$; 8 females; age $=65.82 \pm 7.8$ years; disease duration $=35.64 \pm 15.84$ months $), \quad$ nfv-PPA $\quad(n=19 ; 13$ females; age $=67.42 \pm 8.5$ years; disease duration $=25.89 \pm 12.17$ months $)$, and lv-PPA $(n=17 ; 10$ females; age $=70.71 \pm 7.12$ years; disease duration $=27 \pm 15.58$ months), according to the GornoTempini criteria [4]. Additionally, we included 8 patients who did not meet criteria for any of the three above-mentioned PPA variants, i.e., 3 slowly progressive anarthria cases (i.e., SPA, 1 female; age $=72.33$ \pm 4.73 years; disease duration $=32 \pm 6.93$ months) [24] and 5 mixed PPA patients (i.e., m-PPA, 3 females; age $=66.4 \pm 7.23$ years; disease duration $=28.8 \pm 6.57$ months) [25].

A clinical follow-up of $27.4 \pm 12.55$ months confirmed the diagnosis and assessed the progression of cognitive decline in single patients. See Table 1 for demographic and clinical details.

All subjects, or their informants/caregivers, gave written informed consent to the experimental procedures previously approved by the Ethical Committee of San Raffaele Hospital.

\section{Neuropsychological examination}

At the time of diagnosis and at follow-up, all patients underwent an extensive neuropsychological battery assessing global cognitive status (i.e., MiniMental State Examination), memory (i.e., Digit Span Forward, immediate and delayed recall of Rey Auditory Verbal Learning (RAVLT), recall of copy of the Rey-Osterrieth figure), attention and executive functions (i.e., Attentional Matrices, Raven Colored Progressive Matrices; Digit Span backward), and visuo-spatial abilities (i.e., copy of the Rey-Osterrieth complex figure).

Language examination included (i.e., phonemic (P-F-L)) and semantic (animals-fruits-cars) verbal fluency; the Token test; the naming and the wordpicture matching subtests of the CAGI battery for the assessment of semantic memory [26]; the repetition subtest of the Italian version of the Aachener Aphasia Test (AAT); the grammatical structure (syntax) and sentence comprehension subtests from the "Batteria per l'Analisi dei Deficit Afasici" (BADA) 
[27]. The presence of speech apraxia and articulation difficulties was derived from the medical records.

A careful qualitative study of the spontaneous speech was indeed performed in each case. Slow and hesitant speech with word finding pauses; effortful and halting speech with articulation impairment and distortions; or word-finding impairments with tendency to produce nouns of high frequency, respectively suggesting lv-PPA, nfv-PPA, or sv-PPA syndromes, were considered.

Impaired sentence comprehension and naming, spared single words comprehension, and non-verbal semantics were considered suggestive of lv-PPA and nfv-PPA, while phonological errors and defective repetition were considered the neuropsychological hallmarks of lv-PPA cases [2, 4]. Impaired naming, single words comprehension and non-verbal semantics, and spared repetition were considered the neuropsychological hallmarks of sv-PPA cases $[2,4]$. Impaired digit span task further supports the lv-PPA diagnosis. The presence of speech apraxia, dysarthria, and dysprosodia, accompanied by orofacial and ideomotor apraxia, and mild executive dysfunctions without any global cognitive deterioration characterized the SPA cases [24]. The combination of agrammatism with comprehension deficit, accompanied by poor fluency and frequent paraphasias was considered as suggestive for the m-PPA subtype $[2,25]$.

Standardized measures of socio-emotional abilities (i.e., Ekman-60-Faces Test [28] and Story-based Empathy Task [29]) were also included in the cognitive assessment of a sub-set of PPA patients $(n=18$, $32 \%)$.

\section{FDG-PET imaging acquisition and analysis processing}

FDG-PET scans were performed at baseline for suspected PPA syndrome. The analysis was performed at the Nuclear Medicine Unit, San Raffaele Hospital (Milan, Italy) by physicians expert in FDGPET imaging. FDG-PET acquisitions were done according to the guidelines of the European Association of Nuclear Medicine (EANM), following standardized procedures [30, 31]. Before radiopharmaceutical injection of FDG (185-250 Mbq: usually, $5-8 \mathrm{mCi}$ via a venous cannula), subjects were fasted for at least $6 \mathrm{~h}$ and their blood glucose level was $<120 \mathrm{mg} / \mathrm{dL}$. All images were acquired with a Discovery STE (GE Medical Systems, Milwaukee, WI) multi-ring PET tomography (PET-CT) system (time interval between injection and scan start $=45 \mathrm{~min}$; scan duration $=15 \mathrm{~min}$ ). Images were reconstructed using an ordered subset expectation maximization (OSEM) algorithm. Each PET phase was corrected for attenuation with CT data of the corresponding phase. For each PET scan 47 transaxial tomographic slices of $4.25 \mathrm{~mm}$, re-oriented into the coronal and the sagittal planes, were obtained. The emission images were then reconstructed using a filtered back-projection, using the software provided by the manufacturers. Image processing was performed according to standardized and validated procedures [32-34]. In particular, normalization procedure was performed at the single-subject level to a specific SPM FDG-PET template [32]. At a single-subject level, each patient scan was then tested for relative "hypometabolism" on a voxel-by-voxel basis using a validated procedure that includes comparison with a large normal image database of FDG-PET [33]. Age was included in the two-sample $t$-test analysis as a covariate.

The SPM t-map of hypometabolism resulting from statistical comparison with the normal FDG-PET image database (i.e., one patient versus 112 healthy controls) allowed the definition of disease-specific metabolic patterns. Cerebral regions showing significant hypometabolism were localized using SPM Anatomy toolbox v2.0. The threshold was set at $p=0.05$, FWE-corrected for multiple comparisons at the voxel level. Only clusters containing more than 100 voxels were deemed to be significant.

In addition, in order to evaluate the regional commonalities in the pattern of FDG-PET hypometabolism in each PPA variant, we computed whole-brain group analyses including the cases belonging to sv-PPA $(n=11), n f v-P P A(n=19)$, and lv-PPA $(n=17)$, using a one-sample $t$-test with the contrast images resulting from each first-order "single-subject" analysis. The $p$-value was set at $p<0.001$ uncorrected, with a cluster extent $\mathrm{k}=100$.

\section{Statistical analyses}

Demographic, clinical, and neuropsychological variables were compared among PPA variants. Gender distribution was compared across PPAs using the $\chi^{2}$ test, while one-way ANOVA was used to compare demographic variables. Due to the nonnormal distribution of the neuropsychological data, performances of patients belonging to the different PPA groups were compared using non-parametric statistics. In particular, we used Kruskall-Wallis 
one-way ANOVA and then post-hoc Mann-Whitney tests for pairwise comparison. Statistical analyses were performed on adjusted scores according to normative data [26-29, 35]. Statistical analyses were performed utilizing the IBM SPSS Statistics for Windows version 20.0 (Armonk, NY IBM Corp.) with $\alpha$ set at 0.05 .

In order to infer the probability of a single case of a having or not a specific dementia condition at follow-up after a baseline diagnostic test (i.e., FDGPET scan), we calculated the "Post-test Probability" (i.e., the probability of developing or not developing a specific dementia subtype). Positive Post-test Probability should be conceived as the probability of an individual of developing the condition of interest in the future and not of having the disease. Thus, all FDG-PET SPM t-maps were independently classified by two expert raters (C.C. and D.P.) blinded to baseline and follow-up diagnostic classification. The whole procedure was comparable to that used in a previous study of our group [36]. Inter-rater agreement level between experts resulted in "almost perfect agreement" $(\mathrm{k}=0.85)$. Thus, a single set of classification was selected for the calculation of the "Post-test Probability" value.

Finally, we applied principal component analysis (PCA) to FDG-PET single-subject SPM maps in order to identify clusters of variables highly correlating with each other, with the aim of detecting distinct metabolic patterns predicting the clinical classification. Preliminary, we identified with the Automated Anatomical Labeling (AAL) template the regions of interest (ROIs) considered as the metabolic correlates of language impairments in PPAs. These were the left inferior, middle and superior temporal gyri, temporal pole, superior, middle and inferior frontal gyri, precentral gyrus, and inferior and superior parietal lobule. The relationship between the resulting components of PCA analysis and the classification according to the three main PPA variants [4] was analyzed with the t-student statistics.

\section{RESULTS}

\section{Cognitive profiles of PPA patients}

Although language performances in comprehension (e.g., Token test) and production (e.g., verbal fluency) tasks were impaired in all PPAs, the degree and patterns of errors differed among the three variants. A qualitative analysis of the errors showed that while the nfv-PPA patients had predominant deficits in the comprehension of complex sentences mainly affecting performances on the second part of the Token test, the low scores of sv-PPA patients in this test were mainly due to single word comprehension deficits. In addition, sv-PPA patients also showed reduced scores in naming and word-picture matching subtasks of the CAGI, supporting the selective disruption of semantic memory. Finally, difficulties in the comprehension and repetition of syntactically complex long sentences, and word-finding problems in word generation tasks accounted respectively for the low performances of lv-PPA patients in comprehension and production tasks.

Notably, while sv-PPA was associated with very limited cognitive impairment specifically involving semantic memory, lv-PPA and nfv-PPA variants showed other cognitive impairments in addition to language deficits. Nfv-PPA patients showed reduced attentional abilities and lv-PPA patients impaired verbal short-term memory. Positive behavioral changes (e.g., anxiety, disinhibition, and irritability) were reported by caregivers in, respectively, $64 \%$ and $42 \%$ of sv-PPA and nfv-PPA patients, and only in the $12 \%$ of lv-PPA patients. In contrast, negative behavioral symptoms (i.e., apathy and depression) were equally distributed among all variants and were reported in approximately $50 \%$ of all PPA cases.

Significant differences in the profile of language deficits in the sv-PPA group, compared to both nfv-PPA and lv-PPA groups emerged. In particular, performances in the naming and single-word comprehension tasks were significantly lower in the sv-PPA group $(\mathrm{H}=9.98, p<0.01)$. Although scores on repetition task were higher in this variant compared to the other groups (Table 2), the difference was not significant. The analysis of the non-language cognitive performances showed significantly poorer performances in the lv-PPA and nfv-PPA groups compared to the sv-PPA (Table 2). In particular, a significant impairment in verbal short-term memory (i.e., Digit Span Forward) was found in lv-PPA compared to sv-PPA patients (Mann-Whitney $\mathrm{U}=65, p<0.01$ ). Both nfv-PPA (Mann-Whitney $\mathrm{U}=24, p<0.005$ ) and lv-PPA (Mann-Whitney $\mathrm{U}=33, p<0.01$ ) presented low scores at the Attentional Matrices test compared to the sv-PPA group (Table 2).

All three SPA patients presented a slowly progressive impairment of speech articulation, with and the features of speech apraxia. No other cognitive deficit was reported at the neuropsychological assessment. One SPA showed also mild swallowing difficulties. 
Table 2

Cognitive profiles of the three PPA variants

\begin{tabular}{|c|c|c|c|c|c|}
\hline & sv-PPA $n=11$ & nfv-PPA $n=19$ & lv-PPA $n=17$ & Statistics & Post-hoc \\
\hline Token test (cut-off $=26.25)$ & $24.39 \pm 6.42$ & $21.02 \pm 8.91$ & $23.35 \pm 5.66$ & $\mathrm{H}=0.93$ n.s. & - \\
\hline Phonemic fluency (cut-off $=16$ ) & $11.73 \pm 10.97$ & $12.67 \pm 10.4$ & $15.47 \pm 8.11$ & $\mathrm{H}=1.35$ n.s. & - \\
\hline Semantic fluency $($ cut-off $=24)$ & $12.16 \pm 10.13$ & $19.87 \pm 12.57$ & $20.32 \pm 10.74$ & $\mathrm{H}=3.89$ n.s. & sv-PPA<lv-PPA* \\
\hline $\begin{array}{l}\text { AAT repetition global score } \\
\quad(\text { cut-off }=125 / 150)\end{array}$ & $132.50 \pm 8.7$ & $124.06 \pm 27.01$ & $123.80 \pm 15.71$ & $\mathrm{H}=0.89$ n.s. & - \\
\hline $\begin{array}{l}\text { CAGI naming (cut-off }=46 / 48) \\
\text { sv-PPA }<1 \mathrm{lv}-\mathrm{PPA}\end{array}$ & $16.44 \pm 13.27$ & $34.68 \pm 13.32$ & $33.79 \pm 11.29$ & $\mathrm{H}=9.98^{* *}$ & sv-PPA $<$ nfv-PPA $* *$ \\
\hline $\begin{array}{l}\text { CAGI word-picture matching } \\
\text { (cut-off }=46 / 48)\end{array}$ & $38.44 \pm 10.16$ & $47.10 \pm 2.19$ & $47.74 \pm 0.56$ & $\mathrm{H}=12.9^{* * *}$ & $\mathrm{sv}-\mathrm{PPA}<\mathrm{nfv}-\mathrm{PPA} * *$ \\
\hline \multicolumn{6}{|l|}{$\mathrm{sv}-\mathrm{PPA}<\mathrm{lv}-\mathrm{PPA} * *$} \\
\hline Digit Span (cut-off = 3.5) & $5.26 \pm 1.09$ & $4.37 \pm 1.54$ & $4.15 \pm 0.66$ & $\mathrm{H}=7.18^{*}$ & $1 \mathrm{v}-\mathrm{PPA}<\mathrm{sv}-\mathrm{PPA} * *$ \\
\hline Corsi Span (cut-off = 3.5$)$ & $4.34 \pm 0.64$ & $3.67 \pm 1.02$ & $4.12 \pm 1.32$ & $\mathrm{H}=3.3$ n.s. & - \\
\hline RAVLT immediate recall (cut-off $=28.5$ ) & $21.11 \pm 7.44$ & $24.57 \pm 9.6$ & $23.67 \pm 7.28$ & $\mathrm{H}=1.43$ n.s. & - \\
\hline RAVLT delayed recall (cut-off $=4.68$ ) & $2.57 \pm 2.71$ & $4.8 \pm 2.68$ & $2.87 \pm 3.74$ & $\mathrm{H}=3.06$ n.s. & - \\
\hline $\begin{array}{l}\text { Rey-Osterrieth Complex Figure recall } \\
\quad(\text { cut-off }=9.46)\end{array}$ & $15.10 \pm 7.43$ & $12.66 \pm 6.31$ & $10.35 \pm 4.29$ & $\mathrm{H}=4.52$ n.s. & - \\
\hline $\begin{array}{l}\text { Rey-Osterrieth Complex Figure copy } \\
\quad(\text { cut-off }=28.87)\end{array}$ & $29.90 \pm 4.65$ & $24.03 \pm 8.76$ & $26.59 \pm 8.05$ & $\mathrm{H}=3.12 \mathrm{n} . \mathrm{s}$ & - \\
\hline $\begin{array}{l}\text { Attentive Matrices (cut-off = 30) } \\
\text { lv-PPA<sv-PPA** }\end{array}$ & $43.65 \pm 8.17$ & $28.90 \pm 10.72$ & $34.02 \pm 9.94$ & $\mathrm{H}=11.97^{* * *}$ & nfv-PPA $<$ sv-PPA *** \\
\hline Raven Matrices (cut-off = 17.5) & $27.55 \pm 7.18$ & $22.38 \pm 6.32$ & $25.33 \pm 5.42$ & $\mathrm{H}=4.01$ n.s. & \\
\hline
\end{tabular}

sv-PPA, semantic variant of PPA; nfv-PPA, non fluent variant of PPA; lv-PPA, logopenic variant of PPA; ${ }^{*} p<0.05 ;{ }^{* *} p<0.01 ;{ }^{* *} p<0.005$. Cognitive performances under cut-off are shown in bold.

All the $5 \mathrm{~m}$-PPA cases presented with a mixed phenotype characterized by an impairment of two or more different language components. Core features of lv-PPA (i.e., "impaired single-word retrieval in spontaneous speech and naming" and "impaired repetition of sentences and phrases") and sv-PPA (i.e., "impaired confrontation naming") were present in each m-PPA case. Two out of five m-PPA had also impaired comprehension of syntactically complex sentences.

Socio-emotional recognition and processing deficits were equally present in the main PPA variants (i.e., 3 sv-PPA, 3 nfv-PPA, and 2 lv-PPA). Basic emotion recognition deficits were more frequent (i.e., 8/15 patients with impaired performances on Ek-60-F global score) than disorders of emotion and intention attribution (i.e., SET score) that were found only in sv-PPA patients.

\section{Clinical profiles at the follow-up}

At the clinical follow-up, some PPA patients showed a clinical progression with loss of functional autonomy in the activity of daily living, thus fulfilling criteria for dementia. In details, 6 out of $11 \mathrm{sv-PPA}$ cases progressed to probable fronto-temporal dementia (FTD); 10 out of $17 \mathrm{lv}$-PPA cases to probable Alzheimer's dementia (AD); 11 out of 19 nfv-PPA cases to corticobasal degeneration syndrome (CBDS), and 3 out of $19 \mathrm{nfv}$-PPA to progressive supranuclear palsy (PSP); 1 SPA progressed to CBDS and all $5 \mathrm{~m}$ PPA progressed to dementia (i.e., $2 \mathrm{AD}$ and 3 FTD).

In addition to language impairments, FTD patients showed behavioral disorders and dysexecutive syndrome, while $\mathrm{AD}$ cases verbal and visuo-spatial long-term memory deficits, temporo-spatial disorientation, and apraxia. At the follow-up, CBD and PSP cases presented with parkinsonism and additional clinical signs (i.e., pyramidal sings in CBD and oculomotor disorders in PSP), whereas the neuropsychological evaluation showed attentional and visuo-spatial impairments in $\mathrm{CBD}$ and dysexecutive syndrome in the PSP cases.

The rest of the sample (i.e., 5 sv-PPA, 7 lv-PPA, and $5 \mathrm{nfv}$-PPA and 2 SPA) remained cognitively and functionally stable.

\section{Single-subject FDG-PET SPM profiles}

$$
s v-P P A
$$

In each sv-PPA case the SPM analysis showed significantly reduced glucose metabolism in the temporal pole, middle and inferior temporal gyri, and the insula. While the pattern of regional metabolic changes was relatively consistent across patients, some heterogeneity was evident in the lateralization of hemispheric involvement. Namely, 6 sv-PPA patients out of 11 showed a selective left-sided hypometabolic 
pattern, while in the remaining 5 patients had a bilateral hypometabolism (Table 3A and Fig. 1), more prevalent in the right hemisphere in two patients (i.e., \#8 and \#10). Medial temporal lobe structures (i.e., hippocampal structures and amygdala), the superior temporal and fusiform gyri were involved in $80 \%$ of patients. See Table 3A for further details.

\section{$n f v-P P A$}

The nfv-PPA patients presented the largest heterogeneity in the metabolic patterns. The 5 patients classified at the follow-up as pure nfv-PPA (i.e., patients \#1 to \#5 on Table 3B) showed metabolic decreases in the inferior frontal gyrus, the dorsolateral frontal cortex, the anterior cingulate cortex (ACC), and the insula. In some cases, the superior and inferior parietal cortex was also involved in these pure nfvPPA cases. Hippocampal structures and amygdala were selectively spared in these cases. Those patients classified as $\mathrm{CBD}$ at the clinical-neuropsychological follow-up (i.e., patients \# 6-16 on Table 3B) showed at baseline an asymmetric hypometabolism either in the left or right superior and inferior parietal lobule (n $10 \mathrm{nfv}-\mathrm{PPA} / \mathrm{CBD}$ left-sided hypometabolism, one a right-sided hypometabolism) (Fig. 1). An additional involvement of basal ganglia was present in these cases (Table 3B). The three nfv-PPA patients fulfilling PSP criteria at the follow-up (i.e., patients \# 17-19 on Table 3B) showed at baseline additional involvement of the midbrain and cerebellar hemispheres and a sparing of the parietal cortex.

\section{$l v-P P A$}

In lv-PPA patients, the single-subject FDGPET SPM analysis revealed a rather homogenous hypometabolic pattern mainly involving the left superior, middle and inferior temporal gyri, and the left inferior and superior parietal lobules (Table 3C and Fig. 1). Four lv-PPA patients presented a bilateral hypometabolic pattern (Table 3C). All these cases progressed to $\mathrm{AD}$ dementia within the follow-up time. Hippocampal structures were involved in $65 \%$ of lv-PPA patients.

\section{SPA}

All three SPA patients showed a strictly left hemispheric FDG-PET hypometabolism involving the frontal medial cortex, the insula and the caudate. The SPA case who progressed to CBD at the follow-up showed in addition hypometabolism in the parietal cortex and the thalamus (Table 3D).
$m-P P A$

m-PPA patients progressing to FTD (i.e., \# 6,7,8) showed bilateral fronto-temporal hypometabolism (left > right). One m-PPA case (i.e., \# 5) progressing to $\mathrm{AD}$ showed the typical FDG-PET AD temporoparietal pattern with additional frontal involvement. The other m-PPA patient (i.e., \# 4) who progressed to $\mathrm{AD}$ showed a focal damage of the left inferior frontal gyrus and the anterior regions of temporal lobe as previously reported in mixed-PPA cases [37] (Table 3D).

\section{Commonalities in the FDG-PET SPM patterns across the PPA variants}

The FDG-PET SPM group analysis in sv-PPA patients revealed a predominant left-sided hypometa bolic pattern restricted to the anterior regions of the temporal lobe, the hippocampal structures, and the amygdala. Less extensive involvement of left insula, anterior cingulate, and orbitofrontal cortices also emerged. The nfv-PPA group showed left-sided hypometabolism in the inferior, middle, and superior frontal gyrus, the insula, and the pre-central gyrus, while in the lv-PPA group, the lateral temporal regions, the inferior and superior parietal lobule, and the intraparietal sulcus were hypometabolic. In a few cases, the parietal hypometabolism extended to the right hemisphere. See Fig. 2 for further details on group analyses.

\section{Post-test probability and PCA analyses}

Within the patients diagnosed with a specific dementia subtype at the clinical follow-up (i.e., AD or FTD spectrum) $(n=36)$, the Positive Post-test Probability was $100 \%$. This value indicates the probability of progression to a specific dementia subtype in the single subject after the evidence of a specific hypometabolic pattern at the FDG-PET SPM-t map.

PCA analysis identified three principal components in the left hemisphere: 1) prefrontal cortex including inferior, superior and middle frontal gyri, supplementary motor cortex, and insula, 2) middle and superior temporal gyri, and inferior and superior parietal lobule, and finally 3) anterior regions of temporal lobe. They captured the $75 \%$ of the variance, thus they can be considered as the best dimensional representations of the full data set.

As expected, parametric comparisons between the weights of the single PCA components in the three main PPA variants [4] showed: 1) higher scores 


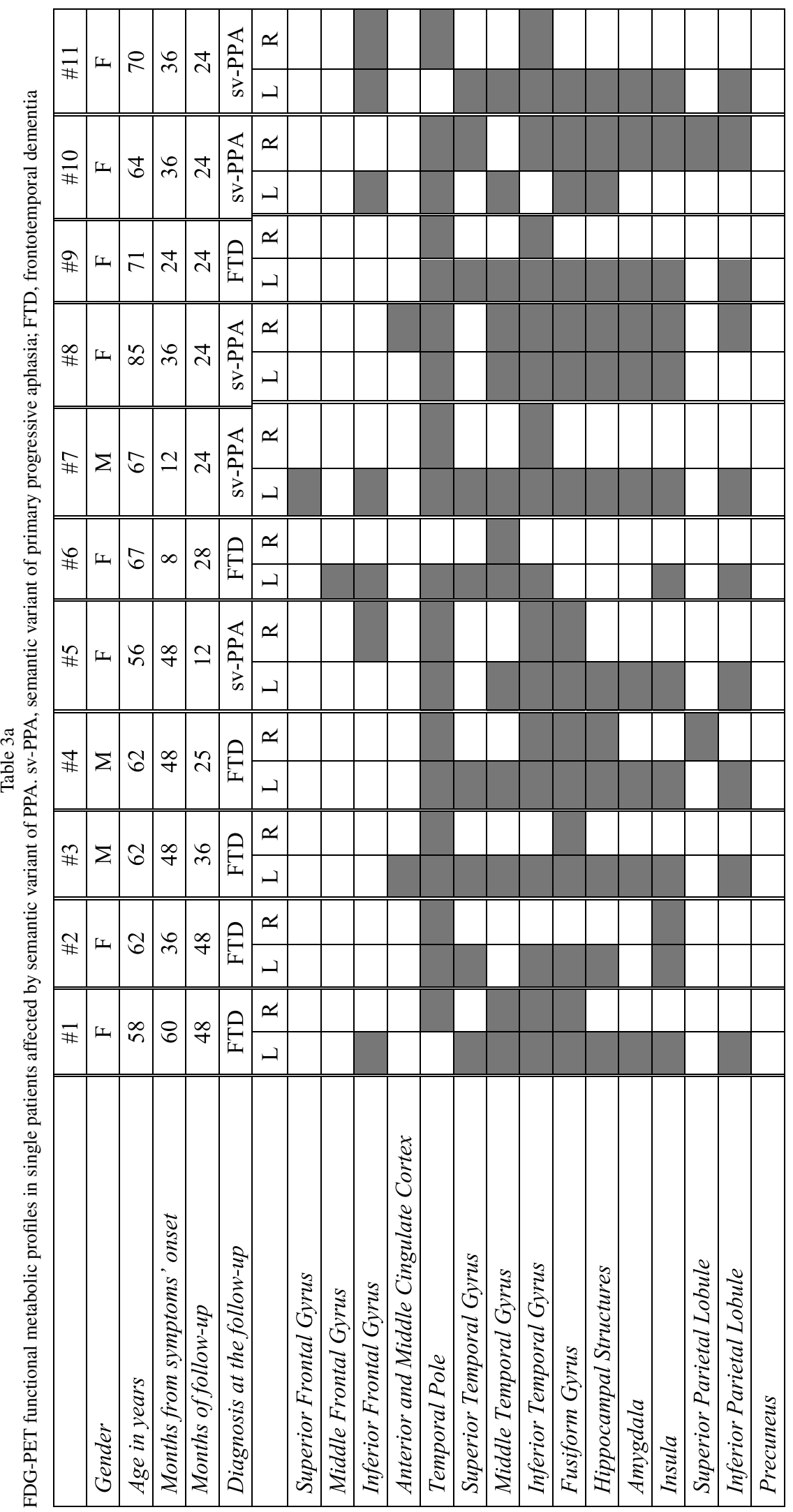




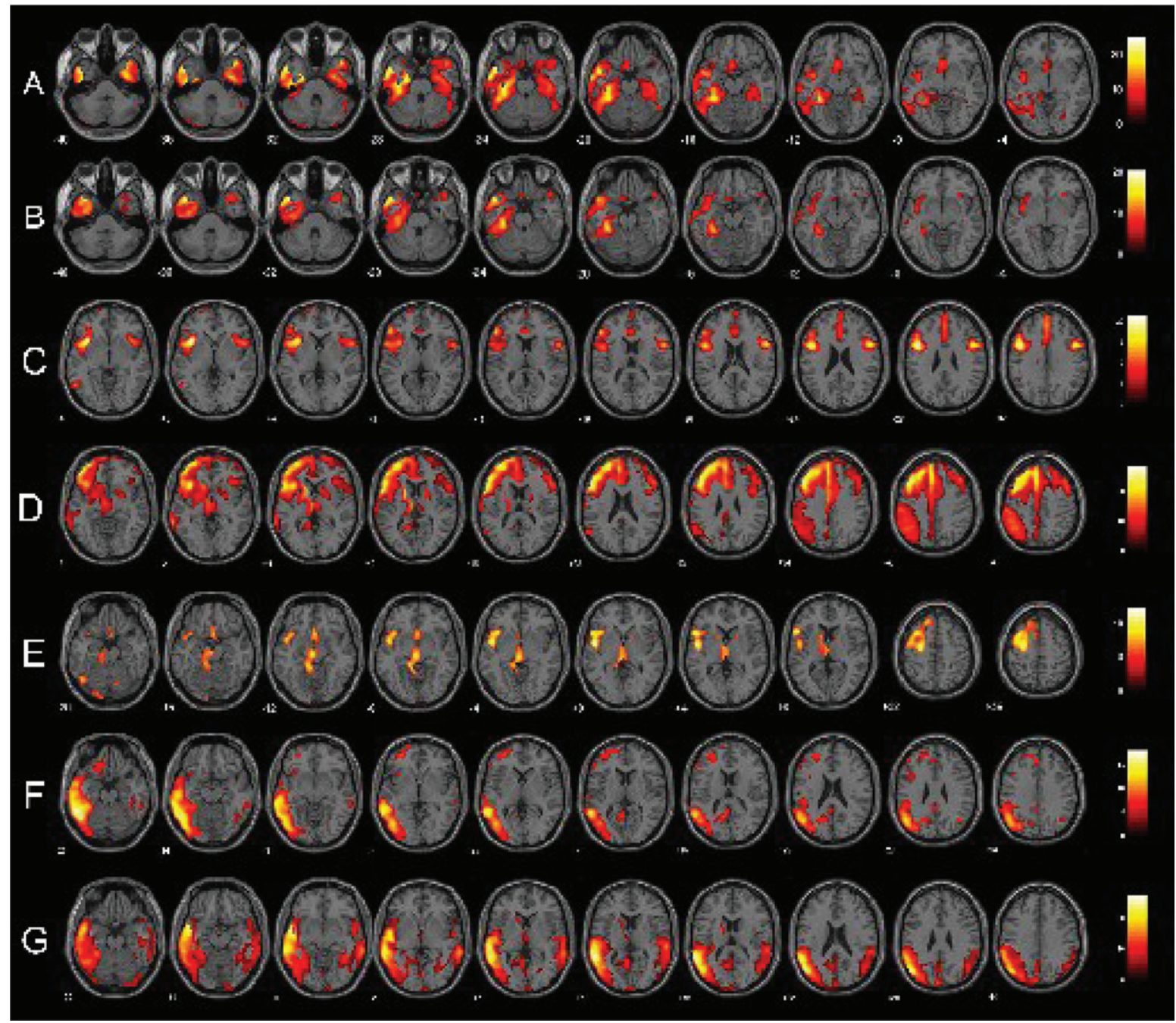

Fig. 1. Single-subject FDG-PET SPM t-maps of PPA patients. Panels A and B show respectively a bilateral and a left-predominant pattern of anterior temporal hypometabolism in two sv-PPA patients. Panels C, D, and E represent respectively patterns of pure nfv-PPA, nfv-PPA classified as CBD and as PSP at the follow-up. Finally, panels F and G show lv-PPA with a left-predominant and a bilateral pattern of hypometabolism. FDG-PET SPM brain hypometabolism patterns are shown in axial view, $p<0.001$ uncorrected.

of the first component in nfv-PPA patients compared to sv-PPA $(\mathrm{t}(27)=2.09, p<0.05)$ and lv-PPA $(\mathrm{t}(33)=2.35, p<0.05)$ patients; 2$)$ higher scores of the second principal component in lv-PPA patients compared to sv-PPA $(\mathrm{t}(26)=4.24, p<0.001)$ and nfv-PPA $(\mathrm{t}(33)=2.707, p<0.05)$ patients; 3 ) higher scores of the third principal component in svPPA patients compared to nfv-PPA $(\mathrm{t}(27)=4.99$; $p<0.001)$ and lv-PPA $(\mathrm{t}(26)=2.69 ; p<0.05)$ patients.

\section{DISCUSSION}

In this study, we assessed FDG-PET metabolic patterns in a large sample of PPA patients at the individual patient level, testing for atypical metabolic patterns and case-to-case variability. FDG-PET scans were analyzed in single subjects with a standardized and validated SPM voxel-based method [32-34]. The results showed different functional metabolic signatures characterizing the PPA variants in single individuals, reflecting different patterns of neurodegeneration, primarily involving language networks, but also non-language brain structures.

In general, brain metabolic patterns were consistent with the current literature focused on group analysis studies (see as example [20]) providing more solid evidence at the single-subject level. As previously demonstrated (e.g., [14, 20, 38]), FDG-PET 


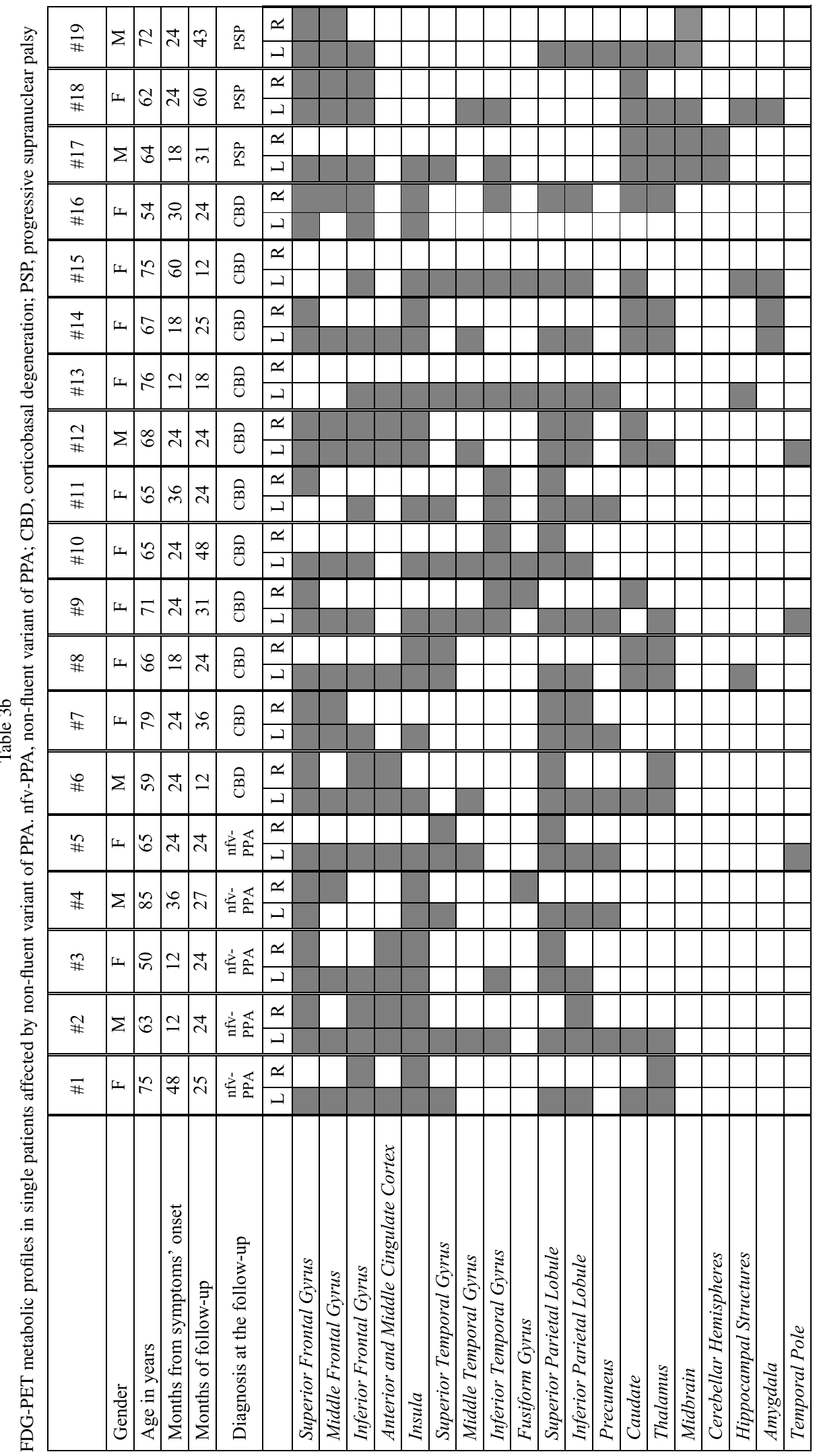




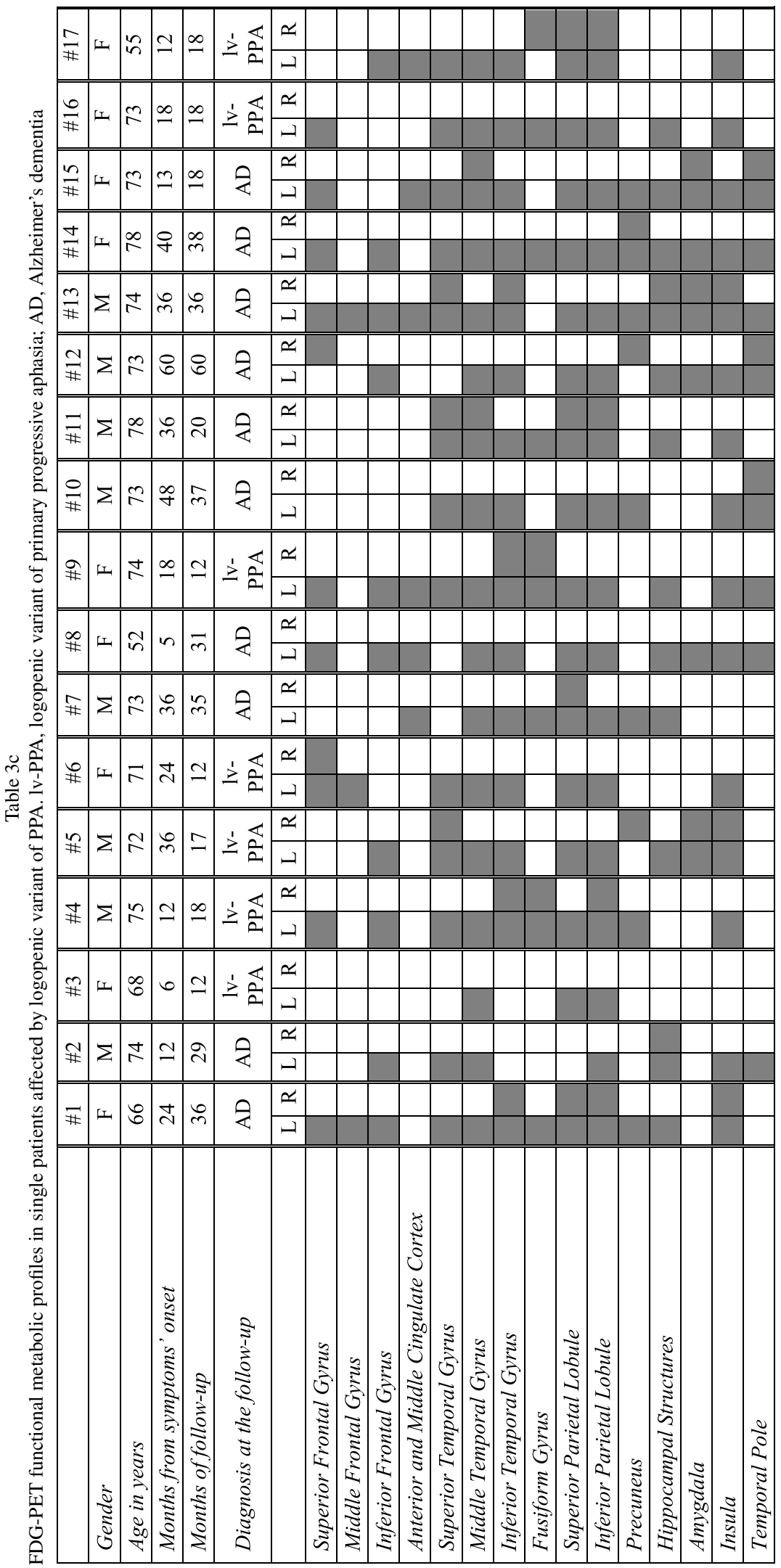


Table 3d

FDG-PET functional metabolic profiles in patients affected by slowly progressive anarthria and mixed PPA. SPA, slowly progressive anarthria; m-PPA, mixed primary progressive aphasia; CBD, corticobasal degeneration; AD, Alzheimer's dementia; FTD, frontotemporal dementia

\begin{tabular}{|c|c|c|c|c|c|c|c|c|c|c|c|c|c|c|c|c|}
\hline & \multicolumn{2}{|c|}{$\# 1$} & \multicolumn{2}{|c|}{$\# 2$} & \multicolumn{2}{|c|}{$\# 3$} & \multicolumn{2}{|c|}{$\# 4$} & \multicolumn{2}{|c|}{$\# 5$} & \multicolumn{2}{|c|}{ \#6 } & \multicolumn{2}{|c|}{ \#7 } & \multicolumn{2}{|c|}{$\# 8$} \\
\hline Gender & \multicolumn{2}{|c|}{ M } & \multicolumn{2}{|c|}{ M } & \multicolumn{2}{|c|}{$\mathrm{F}$} & \multicolumn{2}{|c|}{$\mathrm{F}$} & \multicolumn{2}{|c|}{$\mathrm{F}$} & \multicolumn{2}{|c|}{ M } & \multicolumn{2}{|c|}{$\mathrm{F}$} & \multicolumn{2}{|c|}{$F$} \\
\hline Age in years & \multicolumn{2}{|c|}{76} & \multicolumn{2}{|c|}{74} & \multicolumn{2}{|c|}{67} & \multicolumn{2}{|c|}{77} & \multicolumn{2}{|c|}{58} & \multicolumn{2}{|c|}{66} & \multicolumn{2}{|c|}{62} & \multicolumn{2}{|c|}{69} \\
\hline Months from symptoms' onset & \multicolumn{2}{|c|}{36} & \multicolumn{2}{|c|}{24} & \multicolumn{2}{|c|}{36} & \multicolumn{2}{|c|}{24} & & & & & 2 & & & \\
\hline Months of follow-up & & & & & & & & & & & & & 1 & & & \\
\hline Diagnosis at the baseline & & & & & & & & & & & & & $\mathrm{m}-\mathrm{F}$ & & & PA \\
\hline Diagnosis at the follow-up & & & & & & & & & & & & & FT & & & D \\
\hline & $\mathrm{L}$ & $\mathrm{R}$ & $\mathrm{L}$ & $\mathrm{R}$ & $\mathrm{L}$ & $\mathrm{R}$ & $\mathrm{L}$ & $\mathrm{R}$ & $\mathrm{L}$ & $\mathrm{R}$ & $\mathrm{L}$ & $\mathrm{R}$ & $\mathrm{L}$ & $\mathrm{R}$ & $\mathrm{L}$ & $\mathrm{R}$ \\
\hline Superior Frontal Gyrus & & & & & & & & & & & & & & & & \\
\hline Middle Frontal Gyrus & & & & & & & & & & & & & & & & \\
\hline Inferior Frontal Gyrus & & & & & & & & & & & & & & & & \\
\hline Anterior and Middle Cingulate & & & & & & & & & & & & & & & & \\
\hline Insula & & & & & & & & & & & & & & & & \\
\hline Superior Temporal Gyrus & & & & & & & & & & & & & & & & \\
\hline Middle Temporal Gyrus & & & & & & & & & & & & & & & & \\
\hline Inferior Temporal Gyrus & & & & & & & & & & & & & & & & \\
\hline Fusiform Gyrus & & & & & & & & & & & & & & & & \\
\hline Superior Parietal Lobule & & & & & & & & & & & & & & & & \\
\hline Inferior Parietal Lobule & & & & & & & & & & & & & & & & \\
\hline Precuneus & & & & & & & & & & & & & & & & \\
\hline Caudate & & & & & & & & & & & & & & & & \\
\hline Thalamus & & & & & & & & & & & & & & & & \\
\hline Hippocampal Structures & & & & & & & & & & & & & & & & \\
\hline Amygdala & & & & & & & & & & & & & & & & \\
\hline Temporal Pole & & & & & & & & & & & & & & & & \\
\hline
\end{tabular}

imaging supported an accurate in vivo diagnostic classification at the individual level within the PPA syndromes.

In addition, we found variability in the individual metabolic patterns that revealed possible clinical progression. This was much more evident within the nfv-PPA cases, which in fact may belong to a mixed group of cognitive syndromes (e.g., CBD and PSP) as revealed by the follow-up in our series. In early disease phases, the sparing of non-language domains together with the lack of functional disability is generally considered a main criterion that helps to distinguish PPA from other neurodegenerative disorders, such as the behavioral variant of frontotemporal dementia (bvFTD) or AD. Nevertheless, the clinical picture of PPAs may be sometimes complicated by the presence of non-language cognitive symptoms or neurological signs. PPA syndromes are usually prodromal to more complex clinical pictures belonging to $\mathrm{AD}$ or the FTD spectrum. It represents an intermediate (short- or long-lasting) condition characterized by a relative preservation of the general cognitive functioning with selective impairments of specific language domains. Second-level diagnostic instruments such as our FDG-PET SPM tool may detect early dysfunctional signatures typical of the different dementia conditions even before the clinical appearance of all key symptoms. For example, the hypometabolism in the superior and inferior parietal cortex, which was evident in some nfv-PPA cases, predicted the conversion to $\mathrm{CBD}$ as classified as at the clinical-neuropsychological follow-up (Table 3B and Fig. 1). An involvement of basal ganglia, midbrain, and cerebellum was present in other nfv-PPA cases fulfilling PSP diagnostic criteria at the followup (Table 3B).

Comparably, the pattern of regional dysfunction in lv-PPA might be prognostic for a rapid progression to AD dementia. lv-PPA FDG-PET pattern is indeed largely overlapping with that of $\mathrm{AD}$ [39]. Notably, half lv-PPA cases within our series showed at baseline a bilateral pattern of temporo-parietal 

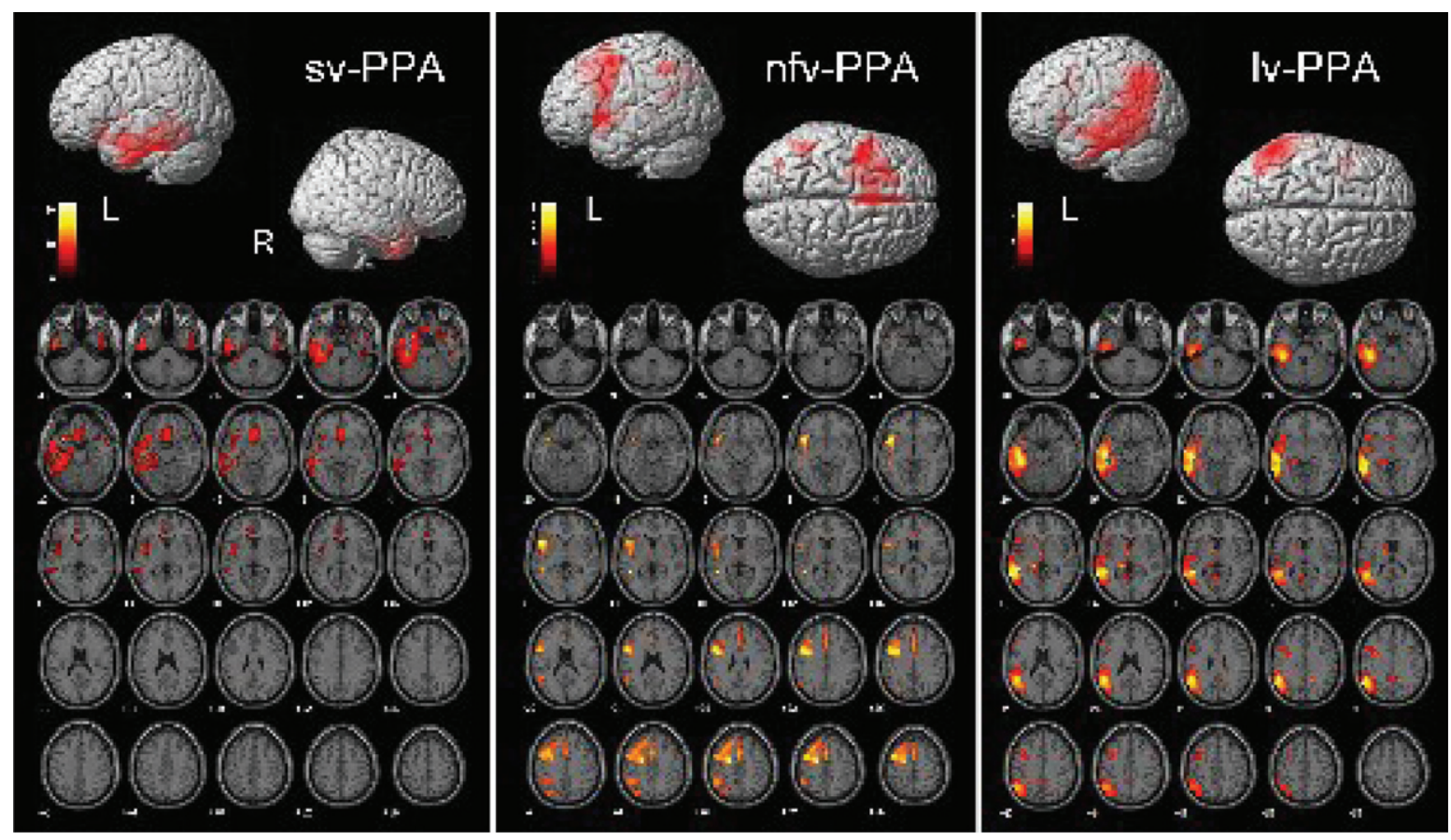

Fig. 2. Brain regions showing significant FDG-PET hypometabolism in the primary progressive aphasia (PPA) variants. On the bottom side of each figure, the FDG-PET hypometabolic pattern resulting from the one-sample SPM group analysis is shown in axial view $(\mathrm{x}=[-40:+56]$; $p<0.001$ uncorrected). On the upper side of each figure, left $(\mathrm{L})$ and right $(\mathrm{R})$ renders are shown. See text for details of the involved brain regions.

dysfunction, undistinguishable from that reported in typical AD. All these lv-PPA cases converted into a full-blown clinical phenotype of AD dementia, with spatial disorientation, apraxia, and episodic amnestic syndrome at the clinical-neuropsychological followup (Table 3C).

In such cases, neuropsychological and functional biomarkers able in early supporting the typical features of each PPA variants and also possible associated phenotypes (i.e., CBD or PSP in nfv-PPA or $\mathrm{AD}$ in lv-PPA) are of extreme value. In this view, as previously suggested in focal dementias [40], the availability of voxel-based reliable methods for the analysis of FDG-PET imaging data at the single-subject level, as the one we applied in this study, can guarantee a more confident diagnosis in PPAs revealing precise regional patterns of reduced brain glucose metabolism in each individual. In fact, in our series, those PPA patients who progressed to dementia condition (i.e., 6 out of 11 Sv-PPA, 10 out of $17 \mathrm{lv}-\mathrm{PPA}, 14$ out of $19 \mathrm{nfv}$ PPA) showed specific FDG-PET patterns suggestive for the specific subtype (i.e., FTD, AD, CBD, and PSP), allowing an early detection of the dementia syndromes and a better prognosis of PPA, and avoiding delay in proper management and therapeutic interventions.

Furthermore, an early accurate prognostic evaluation in PPAs might guarantee a better management of non-language cognitive disorders and any other accompanying symptom in PPAs. For example, a FDG-PET pattern suggestive of PSP may prompt to perform a detailed study of respiratory/swallowing functions, or if suggestive of CBD indicate the need to consider the possible impact of praxic functions. Moreover, it can early address to specific non pharmacological treatments (e.g., speech therapy as well as cognitive stimulation or training of non-language disorders, and psychoeducational support for caregivers of PPA patients with behavioral and socio-emotional disorders), as well as to new pathology-based experimental therapeutic choices.

In addition, we found reduced performances in socio-emotional tasks in all PPA variants, as well as significant metabolic dysfunctions in brain regions implied in social cognitive abilities (e.g., inferior frontal gyrus, right temporal pole, and inferior parietal lobule) [41], in sv-PPA and nfv-PPA individuals. Previous studies showed evidence for a wide spectrum of disorders of socio-emotional processing in 
PPA patients, in particular in sv-PPA [42, 43], but also in nfv-PPA individuals [44]. Although some evidence on the relationship between socio-emotional functioning and reduced brain metabolism has been provided in frontotemporal dementia patients [45, 46], this is not the case of PPAs. The present FDGPET and neuropsychological findings suggest the importance of routinely testing cases suspected for sv-PPA and nfv-PPA also for deficits in social cognition with standardized measures. Future studies are thus encouraged to verify whether PPA-related neurodegenerative processes may cause metabolic changes in the social brain networks.

This study has no pathological confirmation; thus we cannot exclude the possibility of multiple pathologies. It may, however, contribute to the discussion on the use of biomarkers in PPA diagnosis. We strongly support the use of FDG-PET imaging quantitative assessment for the early characterization of PPA variants in single subjects and the use in clinical setting of such methods for a better diagnostic and prognostic definition of possible endo-phenotypes. The early detection of specific dysfunctional patterns might easily predict a possible conversion to a more complex clinical syndromes or dementia conditions.

\section{ACKNOWLEDGMENTS}

We thank Dr. Maria Cristina Giusti, Michele Zamboni, and Valeria Golzi for patients' recruitment.

Dr. Cerami was funded by Fondazione Eli-Lilly (Eli-Lilly grant 2011 "Imaging of neuroinflammation and neurodegeneration in prodromal and presymptomatic Alzheimer's disease phases"). This work has been partially supported by EU FP7 INMIND Project (FP7-HEALTH-2011, grant agreement no. 278850).

Authors' disclosures available online (http://j-alz. com/manuscript-disclosures/16-0682r1).

\section{REFERENCES}

[1] Mesulam MM, Weintraub S (1992) Spectrum of primary progressive aphasia. Baillieres Clin Neurol 1, 583-609.

[2] Cerami C, Cappa SF (2016) Primary progressive aphasia. In: Hodges' Frontotemporal Dementia, 2nd ed. Cambridge University Press.

[3] Harris JM, Gall C, Thompson JC, Richardson AM, Neary D, du Plessis D, Pal P, Mann DM, Snowden JS, Jones M (2013) Classification and pathology of primary progressive aphasia. Neurology 81, 1832-1839.

[4] Gorno-Tempini ML, Hillis AE, Weintraub S, Kertesz A, Mendez M, Cappa SF, Ogar JM, Rohrer JD, Black S, Boeve BF, Manes F, Dronkers NF, Vandenberghe R, Rascovsky K, Patterson K, Miller BL, Knopman DS, Hodges JR, Mesulam
MM, Grossman M (2011) Classification of primary progressive aphasia and its variants. Neurology 76, 1006-1014.

[5] Gorno-Tempini ML, Dronkers NF, Rankin KP, Ogar JM, Phengrasamy L, Rosen HJ, Johnson JK, Weiner MW, Miller BL (2004) Cognition and anatomy in three variants of primary progressive aphasia. Ann Neurol 55, 335-346.

[6] Josephs KA, Duffy JR, Strand EA, Machulda MM, Senjem ML, Master AV, Lowe VJ, Jack CR Jr, Whitwell JL (2012) Characterizing a neurodegenerative syndrome: Primary progressive apraxia of speech. Brain 135(Pt 5) 1522-1536.

[7] Perez DL, Dickerson BC, McGinnis SM, Sapolsky D, Johnson K, Searl M, Daffner KR (2013) You don't say: Dynamic aphasia, another variant of primary progressive aphasia? J Alzheimers Dis 34, 139-144.

[8] Mesulam M, Weintraub S, Rogalski EJ, Wieneke C, Geula C, Bigio EH (2014) Asymmetry and heterogeneity of Alzheimer's and frontotemporal pathology in primary progressive aphasia. Brain 137, 1176-1192.

[9] Diehl J, Grimmer T, Drzezga A, Riemenschneider M, Förstl H, Kurz A (2004) Cerebral metabolic patterns at early stages of frontotemporal dementia and semantic dementia. A PET study. Neurobiol Aging 25, 1051-1056.

[10] Nestor PJ, Fryer TD, Hodges JR (2006) Declarative memory impairments in Alzheimer's disease and semantic dementia. Neuroimage 30, 1010-1020.

[11] Acosta-Cabronero J, Patterson K, Fryer TD, Hodges JR, Pengas G, Williams GB, Nestor PJ (2011) Atrophy, hypometabolism and white matter abnormalities in semantic dementia tell a coherent story. Brain 134(Pt 7), 2025-2035.

[12] Iaccarino L, Crespi C, Della Rosa PA, Catricalá E, Guidi L, Marcone A, Tagliavini F, Magnani G, Cappa SF, Perani D (2015) The semantic variant of primary progressive aphasia: Clinical and neuroimaging evidence in single subjects. $P L O S$ One 10, e0120197.

[13] Nestor PJ, Graham NL, Fryer TD, Williams GB, Patterson K, Hodges JR (2003) Progressive non-fluent aphasia is associated with hypometabolism centred on the left anterior insula. Brain 126(Pt 11), 2406-2418.

[14] Rabinovici GD, Jagust WJ, Furst AJ, Ogar JM, Racine CA, Mormino EC, O’Neil JP, Lal RA, Dronkers NF, Miller BL, Gorno-Tempini ML (2008) Abeta amyloid and glucose metabolism in three variants of primary progressive aphasia. Ann Neurol 64, 388-401.

[15] Matías-Guiu JA, Cabrera-Martín MN, Moreno-Ramos T, Valles-Salgado M, Fernandez-Matarrubia M, Carreras JL, Matías-Guiu J (2015) Amyloid and FDG-PET study of logopenic primary progressive aphasia: Evidence for the existence of two subtypes. J Neurol 262, 1463-1472.

[16] Desgranges B, Matuszewski V, Piolino P, Chételat G, Mézenge F, Landeau B, de la Sayette V, Belliard S, Eustache F (2007) Anatomical and functional alterations in semantic dementia: A voxel-based MRI and PET study. Neurobiol Aging 28, 1904-1913.

[17] Kim EJ, Kim BC, Kim SJ, Jung DS, Sin JS, Yoon YJ, Chin J, Lee KH, Na DL (2012) Clinical staging of semantic dementia in an FDG-PET study using FTLD-CDR. Dement Geriatr Cogn Disord 34, 300-306.

[18] Drzezga A, Grimmer T, Henriksen G, Stangier I, Perneczky R, Diehl-Schmid J, Mathis CA, Klunk WE, Price J, DeKosky S, Wester HJ, Schwaiger M, Kurz A (2008) Imaging of amyloid plaques and cerebral glucose metabolism in semantic dementia and Alzheimer's disease. Neuroimage 39, 619-633.

[19] Mion M, Patterson K, Acosta-Cabronero J, Pengas G, Izquierdo-Garcia D, Hong YT, Fryer TD, Williams GB, 
Hodges JR, Nestor PJ (2010) What the left and right anterior fusiform gyri tell us about semantic memory. Brain 133, 3256-3268.

[20] Matias-Guiu JA, Cabrera-Martín MN, Moreno-Ramos T, García-Ramos R, Porta-Etessam J, Carreras JL, MatíasGuiu J (2015) Clinical course of primary progressive aphasia: Clinical and FDG-PET patterns. J Neurol 262, 570-577.

[21] Whitwell JL, Duffy JR, Strand EA, Machulda MM, Senjem ML, Schwarz CG, Reid R, Baker MC, Perkerson RB, Lowe VJ, Rademakers R, Jack CR Jr, Josephs KA (2015) Clinical and neuroimaging biomarkers of amyloid-negative logopenic primary progressive aphasia. Brain Lang 142, 45-53.

[22] Josephs KA, Duffy JR, Fossett TR, Strand EA, Claassen DO, Whitwell JL, Peller PJ (2010) Fluorodeoxyglucose F18 positron emission tomography in progressive apraxia of speech and primary progressive aphasia variants. Arch Neurol 67, 596-605.

[23] Cerami C, Marcone A, Galimberti D, Villa C, Fenoglio C, Scarpini E, Cappa SF (2013) Novel missense progranulin gene mutation associated with the semantic variant of primary progressive aphasia. J Alzheimers Dis 36, 415-420.

[24] Broussolle E, Bakchine S, Tommasi M, Laurent B, Bazin B, Cinotti L, Cohen L, Chazot G (1996) Slowly progressive anarthria with late anterior opercular syndrome: A variant form of frontal cortical atrophy syndromes. J Neurol Sci 144, 44-58.

[25] Mesulam M, Wieneke C, Rogalski E, Cobia D, Thompson C, Weintraub S (2009) Quantitative template for subtyping primary progressive aphasia. Arch Neurol 66, 1545-1551.

[26] Catricalá E, Della Rosa PA, Ginex V, Mussetti Z, Plebani V, Cappa SF (2013) An Italian battery for the assessment of semantic memory disorders. Neurol Sci 34, 985-993.

[27] Miceli G, Laudanna A, Capasso R (2006) Batteria per l'analisi dei deficit afasici (B.A.D.A.). Versione riveduta e computerizzata su CD-ROM. Bologna E.M.S. ID: 26851.

[28] Dodich A, Cerami C, Canessa N, Crespi C, Marcone A, Arpone M, Realmuto S, Cappa SF (2014) Emotion recognition from facial expressions: A normative study of the Ekman 60-Faces Test in the Italian population. Neurol Sci 35, 1015-1021.

[29] Dodich A, Cerami C, Canessa N, Crespi C, Iannaccone S, Marcone A, Realmuto S, Lettieri G, Perani D, Cappa SF (2015) A novel task assessing intention and emotion attribution: Italian standardization and normative data of the Story-based Empathy Task. Neurol Sci 36, 1907-1912.

[30] Morbelli S, Drzezga A, Perneczky R, Frisoni GB, Caroli A, van Berckel BN, Ossenkoppele R, Guedj E, Didic M, Brugnolo A, Sambuceti G, Pagani M, Salmon E, Nobili F (2012) Resting metabolic connectivity in prodromal Alzheimer's disease. A European Alzheimer Disease Consortium (EADC) project. Neurobiol Aging 33, 2533-2550.

[31] Varrone A, Asenbaum S, Vander Borght T, Booij J, Nobili F, Någren K, Darcourt J, Kapucu OL, Tatsch K, Bartenstein P, Van Laere K, European Association of Nuclear Medicine Neuroimaging Committee (2009) EANM procedure guidelines for PET brain imaging using [18F]FDG, version 2. Eur J Nucl Med Mol Imaging 36, 2103-2110.

[32] Della Rosa PA, Cerami C, Gallivanone F, Prestia A, Caroli A, Castiglioni I, Gilardi MC, Frisoni G, Friston K, Ashburner J, Perani D, Consortium EADC-PET (2014)
A standardized [18F]-FDG-PET template for spatial normalization in statistical parametric mapping of dementia. Neuroinformatics 12, 575-593.

[33] Perani D, Della Rosa PA, Cerami C, Gallivanone F, Fallanca F, Vanoli EG, Panzacchi A, Nobili F, Pappatá S, Marcone A, Garibotto V, Castiglioni I, Magnani G, Cappa SF, Gianolli L, Consortium EADC-PET (2014) Validation of an optimized SPM procedure for FDG-PET in dementia diagnosis in a clinical setting. Neuroimage Clin 6, 445-454.

[34] Perani D, Cerami C, Caminiti SP, Santangelo R, Coppi E, Ferrari L, Pinto P, Passerini G, Falini A, Iannaccone S, Cappa SF, Comi G, Gianolli L, Magnani G (2016) Crossvalidation of biomarkers for the early differential diagnosis and prognosis of dementia in a clinical setting. Eur J Nucl Med Mol Imaging 43, 499-508.

[35] Spinnler H, Tognoni G (1987) Standardizzazione e taratura italiana di test neuropsicologici. Masson Italia Periodici.

[36] Cerami C, Della Rosa PA, Magnani G, Santangelo R, Marcone A, Cappa SF, Perani D (2014) Brain metabolic maps in mild cognitive impairment predict heterogenity of progression to dementia. Neuroimage Clin 7, 187-194.

[37] Mesulam MM, Wieneke C, Thompson C, Rogalski E, Weintraub S (2012) Quantitative classification of primary progressive aphasia at early and mild impairment stages. Brain 135(Pt 5), 1537-1553.

[38] Matias-Guiu JA, Cabrera-Martín MN, García-Ramos R, Moreno-Ramos T, Valles-Salgado M, Carreras JL, MatiasGuiu J (2014) Evaluation of the new consensus criteria for the diagnosis of primary progressive aphasia using fluorodeoxyglucose positron emission tomography. Dement Geriatr Cogn Disord 38, 147-152.

[39] Perani D (2013) FDG PET and cognitive symptoms of dementia. Clin Transl Imaging 1, 247-260.

[40] Taswell C, Yates P, Shimada H, Leyton CE, Ballard KJ, Piguet O, Burrell JR, Hodges JR, Rowe CC (2015) 18FFDG PET improves diagnosis in patients with focal-onset dementias. J Nucl Med 56, 1547-1553.

[41] Van Overwalle F (2009) Social cognition and the brain: A meta-analysis. Hum Brain Mapp 30, 829-858.

[42] Duval C, Bejanin A, Piolino P, Laisney M, de La Sayette V, Belliard S, Eustache F, Desgranges B (2012) Theory of mind impairments in patients with semantic dementia. Brain 135(Pt 1), 228-241.

[43] Kumfor F, Piguet O (2012) Disturbance of emotion processing in frontotemporal dementia: A synthesis of cognitive and neuroimaging findings. Neuropsychol Rev 22, 280-297.

[44] Couto B, Manes F, Montañés P, Matallana D, Reyes P, Velasquez M, Yoris A, Baez S, Ibáñez A (2013) Structural neuroimaging of social cognition in progressive non-fluent aphasia and behavioral variant of frontotemporal dementia. Front Hum Neurosci 7, 467.

[45] Cerami C, Dodich A, Iannaccone S, Marcone A, Lettieri G, Crespi C, Gianolli L, Cappa SF, Perani D (2015) Right limbic FDG-PET hypometabolism correlates with emotion recognition and attribution in probable behavioral variant of frontotemporal dementia patients. PLoS One 10, e0141672.

[46] Le Bouc R, Lenfant P, Delbeuck X, Ravasi L, Lebert F, Semah F, Pasquier F (2012) My belief or yours? Differential theory of mind deficits in frontotemporal dementia and Alzheimer's disease. Brain 135(Pt 10), 3026-3038. 